\title{
About the Ontology of Quantum Chemistry
}

\section{Sobre la ontología de la química cuántica}

\author{
Juan Camilo Martínez González \\ Universidad de Buenos Aires/CONICET \\ Argentina \\ olimac62@hotmail.com \\ https://orcid.org/0000-0002-7239-1763
}

Recibido: 03 - $04-2018$.

Aceptado: 20 - 06 - 2018.

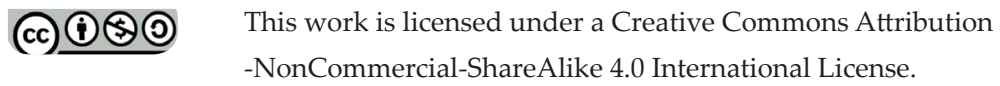




\begin{abstract}
Quantum chemistry is the branch of chemistry whose primary focus is the application of quantum mechanics to chemical systems at the molecular level. Precisely due to its peculiar position between chemistry and physics, in the last times it has begun to engage the interest of the philosophers of chemistry. Nevertheless, in this philosophical field, quantum chemistry has been studied mainly from a historical viewpoint or from a perspective interested on methodological issues. By contrast, the question that will guide this article is: what kind of ontic items are those studied by quantum chemistry? In order to develop the argumentation, first the relevance of the ontological questions will be addressed. Then, it will be considered in what measure the Born-Oppenheimer approximation and the quantum-chemistry concept of electron fit in the quantum theoretical context. Finally, some issues about what quantum chemistry refers to will be discussed.
\end{abstract}

Key words: quantum mechanics; indistinguishability; non-separability; contextuality; quantum chemistry.

\title{
Resumen
}

Química cuántica es la rama de la química que se ocupa principalmente de la aplicación de la mecánica cuántica a los sistemas químicos a un nivel molecular. Gracias a su peculiar posición entre la química y la física, ha comenzado a ser una disciplina de interés para los filósofos de la química. Sin embargo, en el ámbito filosófico, la química cuántica se ha estudiado principalmente, desde una perspectiva histórica o desde cuestiones metodológicas. En contraste, la pregunta que guía este artículo es: ¿qué clase de entidades son estudiadas por la química cuántica? Para desarrollar mi argumento, discutiré, en primer lugar, la importancia de las preguntas ontológicas. Después, consideraré en qué medida la aproximación BornOppenheimer y el concepto de electrón de la química cuántica se ajustan al contexto teórico cuántico. Finalmente, discutiré algunas cuestiones acerca de a qué se refiere la química cuántica.

Palabras clave: mecánica cuántica; indistinguibilidad; no separabilidad; contextualidad; química cuántica. 


\section{Introduction}

As usually pointed out, the philosophy of science of the twentieth century was led by physics: scientific problems were treated following the model of physical problems. It was just in the last decades of the century that the philosophies of certain particular sciences, such as biology and chemistry, began to emerge as autonomous fields of research. However, whereas the philosophy of biology established its specific agenda of research from the very beginning, chemistry could not cut completely its ties with physics: due to the closeness between the two disciplines, one of the hottest topics in the philosophy of chemistry since its beginning and till now is the relationship between chemistry and physics, in particular, quantum mechanics. In this context, quantum chemistry acquires a particular relevance.

Quantum chemistry is the branch of chemistry whose primary focus is the application of quantum mechanics to chemical systems at the molecular level. Precisely due to its peculiar position between chemistry and physics, in the last times it has begun to engage the interest of the philosophers of chemistry. Nevertheless, in this philosophical field, quantum chemistry has been studied mainly from a historical viewpoint (Simões and Gavroglu, 2001; Gavroglu and Simões, 2012) or from a perspective interested on methodological issues (Scerri, 2004; Hettema 2009, 2012; Accorinti \& Martínez González, 2016). In the present article, I will let aside those approaches to focus on ontological matters. The question that will guide the work is: what kind of ontic items are those studied by quantum chemistry? In order to develop the arguments, this work will be organized as follows. In Section 2, the relevance of the ontological questions will be addressed. Section 3 will be devoted to considering the Born-Oppenheimer approximation and in what measure it fits in the quantum theoretical context. In Section 4 , the ontological category of electrons will be discussed in light of the conceptual peculiarities of quantum mechanics. Finally, in Section 5 I will draw some conclusions regarding what ontology quantum chemistry refers to. 


\section{The relevance of the ontological questions}

Since its inception, the Western philosophical thought was interested in the constitution of reality: what the fundamental entities of nature are and how their manifest behavior arises. Although this attitude decayed during the first part of Medieval times, at the end of the Middle Ages and notably in the Renaissance, the interest in the knowledge of nature reborn with new strength and persisted when the different scientific disciplines branched off from philosophy during the following centuries. The idea that science describes reality dominated science during most of its history; nevertheless, for the philosophy of science of the twentieth century, that idea began to move to the background. In particular, the logical positivism coined by the Vienna Circle during the first decades of the century developed a strong and explicit rejection of metaphysics and, as a consequence, claimed that the only legitimate task of philosophy is to study and clarify the language of science. This stance exerted a great influence on the philosophical world, giving rise to the so-called 'linguistic turn' of the analytical philosophy, which dominated the discipline in the Anglophone academy. According to this position, the traditional themes of philosophy must be addressed from the viewpoint of language; in the philosophy of science, this led to focus on methodological issues about theories, explanation, intertheory relations, etc., ignoring or even rejecting ontological matters. It was necessary to wait until the last decades of the 20th century to witness a renewed interest in ontological matters: philosophers of science began to discuss not only about scientific theories, but mainly about the nature described by those theories.

The interest in ontology enters the philosophy of chemistry recently. In particular, the efforts to elucidate the ontological categories in chemistry have generally referred to macroscopic substance related chemistry (cf., for example, van Brakel (2011), Harré (2009)), but without any interest in the ontological questions about quantum chemistry. For instance, Joachim Schummer (2008) stresses the difference between chemical ontology and the physical ontology in terms of the opposition between matter and form. According to the author, from the form perspective the essential properties of bodies are intrinsic geometrical properties, such as size and shape; consequently, change is only motion 
in space. The matter-perspective, on the contrary, is interested in the composition of bodies, the particular materials that each body consists of; the properties that describe the world are dispositions, that is, they describe the behavior of an object under certain conditions; so, change is manifested as reaction and transmutation. In turn, Lucía Lewowicz and Olimpia Lombardi (2013) prefer to stress the difference between the categories that underlie the chemical and the physical domains: the physical world is an individual-and-properties ontology, whereas the chemical world is a stuff ontology. Individual and stuff are different ontological categories, referred to by different types of terms: count terms and mass terms. An individual is a whole unity in the sense that, as such, it is indivisible: this means that either it cannot be divided or, if it can, the results of the division are individuals or parts different from the original one. Individuals are either one (each one of them) or many, that is, a plurality. In the plural case, individuals form aggregates, where they preserve their identity and can be counted. Unlike individuals, a portion of stuff can be divided into portions of the same stuff, that is, it can be divided without losing its identity. In spite of the fact that portions of stuff are multiple, they do not behave as individuals. In fact, if portions of stuff are put together in an aggregate, they cannot be counted: the aggregate of two portions of water is not "two waters" but "more water". In turn, portions of stuff cannot be reidentified once they are put together: it cannot be said that "this" is one and "that" is the other of the original portions of water.

Besides those two ontological categories, Lewowicz and Lombardi (2013) consider the relation between the ontology of stuff, corresponding to the macroscopic chemistry of substances, and the ontology of individuals, corresponding to molecular chemistry. The authors point out how the deep breakdown between the two categories represents an obstacle to traditional ontological reductionism in chemistry, according to which macroscopic chemical phenomena and chemical entities are, when considered in depth, nothing else than molecular entities and processes. When that breakdown is acknowledged, supposing that stuffs are nothing else than collections of minute individuals is no longer acceptable.

The analyses offered by Schummer and by Lewowicz and Lombardi are confined to the relation between macro-chemistry and molecular chemistry. However, analogous ontological questions can be posed regarding the links between the ontology of molecular chemistry and 
the underlying quantum ontology. For instance, in several works Robin Hendry $(2004,2008,2010)$ has stressed the impossibility to reduce molecular structure to quantum mechanics. From a Kantian rooted position, Olimpia Lombardi and Martín Labarca $(2005,2006)$ advocate for the autonomy of the chemical world by emphasizing, not only the non-reducibility of molecular chemistry to quantum mechanics, but also the conceptual discontinuity between the two theories (Labarca \& Lombardi, 2010; see also Lombardi, 2014).

The two cases considered above, macroscopic chemistry versus molecular chemistry and molecular chemistry versus quantum mechanics, bring to light ontological breakdowns that obstacle the uncritically assumed reducibility of one of the ontic domains to the other. Nevertheless, those breakdowns do not hinder the possibility of a coherent ontological view of each one of the three involved domains. In other words, the discontinuities just mentioned affect inter-domain relationships, but not intra-domain matters. This is precisely the difference with respect to quantum chemistry, where different, even incompatible scientific theories converge to the same discipline. In this case, the problem is not to find the links between different ontic domains, but rather to assess whether a consistent ontology for quantum chemistry is possible at all.

\section{The Born-Oppenheimer approximation}

Proposed in the early days of quantum mechanics by Max Born and Robert Oppenheimer (1927), the Born-Oppenheimer approximation (BOA) is still an indispensable tool in quantum chemistry by separating the quantum wave function of the molecule into its electronic and its nuclear components. This separation implies treating the nuclei as classical-like particles at rest in definite positions: they supply a classical nuclear framework that produces the electrical field where electrons move. The geometrical disposition of the nuclei defines molecular structure, and it is an indispensable and unquestioned ingredient of quantum chemistry.

The critical step of the BOA is the so-called 'clamped nuclei approximation': electrons are conceived as moving in the Coulomb potential produced by nuclei at rest, "clamped" at definite positions. This move is justified on the basis of intuitions coming from classical physics: under the assumption that the nuclear mass is much greater 
than the electronic mass, $M>>m$, the kinetic energy of the nuclei, $T_{n}=P_{n}^{2} / 2 M$, can be neglected when compared with the kinetic energy of the electrons $T_{e}=P_{e}^{2} / 2 \mathrm{~m}$. From a classical viewpoint, if a particle has zero kinetic energy, it is at rest at a definite position. However, this is not a classical domain, but a quantum theoretical framework where, as it is well known, classical intuitions frequently do not work (for a detailed analysis of the assumptions underlying the BOA, see Lombardi \& Castagnino, 2010).

Let us consider what kind or approximation underlies the BornOppenheimer strategy. In the philosophy of science literature, it is usual to distinguish between factual and counterfactual limits (Bruer, 1982; Rohrlich, 1989). For instance, classical mechanics arises from special relativity by the application of the factual limit $v / c \rightarrow 0$, that is, an approximation for velocities $v$ much lower than the speed of light $c$, or by letting $c \rightarrow \infty$, that is, a counterfactual limit given that $c$ has a finite value in the relativistic context. Another case is the classical limit of quantum mechanics, which involves the factual limit $\hbar / S \rightarrow 0$, representing situations in which the action $S$ is much higher that the quantum of action $\hbar$, but that can also be based on the counterfactual limit $\hbar \rightarrow 0$. It is interesting to notice that a counterfactual limit is legitimate only if it can be replaced by a factual limit. In the case of the $\mathrm{BOA}$, the great difference between the masses of nuclei and electrons, $M \gg m$, is formally introduced by means of the limit $m / M \rightarrow 0$. But, what kind of limit is $m / M \rightarrow 0$ ? The limit $m \rightarrow 0$ is counterfactual because the mass of the electron is a constant, and the limit $M \rightarrow \infty$ is also counterfactual because, since the masses of the nuclei's components are also constant, it would require an infinite number of components of the nuclei. By contrast, the limit $m / M \rightarrow 0$ is usually conceived as a factual, "practical" limit that expresses the high difference between the two masses.

However, the limit $m / M \rightarrow 0$ is also counterfactual, because it leads to results that are inconsistent with the theory on which it is applied. Let us consider, in the framework of classical mechanics, the friction on a body in motion on a surface: one may suppose that the friction is zero. The assumption is likely false because surfaces perfectly smooth surely do not exist in the real world. However, the assumption 
is compatible with classical mechanics: the existence of such a surface would not contradict the postulates of the theory. The situation is completely different if one supposes, in the context of special relativity, that two spatially separated objects interact instantaneously: this assumption contradicts one of the postulates of the theory, in particular, that which fixes $c$ as the speed limit of any physical interaction. The limit $m / M \rightarrow 0$ belongs to the second kind, since it leads to a contradiction with the Heisenberg principle, according to which no quantum particle can simultaneously have definite values of position and of momentum. As Hasok Chang clearly states:

In this «clamping-down» approximation, the atomic nuclei are treated essentially as classical particles; (...) this picture is non-quantum in a very fundamental way as the simultaneous assignment of fixed positions and fixed momenta (namely, zero) to them violates the Heisenberg uncertainty principle (Chang, 2015, p. 198).

Hendry $(1998,2010)$ points out that the proxy defense of BornOppenheimer models assumes that using them instead of the exact solution makes only a small difference to the energy. However, from a theoretical viewpoint, those models simply assume the facts about molecular structure that ought to be explained (Hendry, 2010, p. 186). The above arguments show that the BOA, with its clamped nuclear assumption, is not a mere innocent approximation that admits a proxy defense in a reductionist context. It not only puts molecular structure by hand in a way that cannot be derived from quantum mechanics. What the approximation adds to quantum mechanics contradicts the very principles of the theory.

On the other hand, it is interesting to note that, during the last decade, some approximate methods that intend to go "beyond" the Born-Oppenheimer approach have been introduced in chemistry (see, for example, Diestle, 2013). Those methods, when applicable, give good results for the calculation of the molecular energy levels. However, when they focus on molecular dynamics, they are still based on semiclassical approaches, which confront the widely discussed problem of the classical limit of quantum mechanics and, as a consequence, do not clearly account for the structure of a molecule in quantum terms. 


\section{The ontological category of electrons}

As a corollary of the previous section it might be concluded that the classical features sweep into quantum chemistry only regarding nuclei through the BOA. In fact, electrons are particles to which the Schrödinger equation, a paradigmatic quantum element, applies. However, the situation is not so simple, because in many cases the electrons in the molecule, as conceived in quantum chemistry, do not have the specific features of quantum systems: contextuality, non-separability and indistinguishability.

\subsection{Contextuality}

Originally, the Heisenberg principle was endowed with an epistemic reading, manifested by its very name, 'uncertainty principle': the particle is in some determinate position, but we do not know which one. This reading was already implicit in the so called 'Heisenberg microscope', a thought experiment proposed by Werner Heisenberg (1930) himself, according to which the measurement of the position of the particle disturbs its momentum and vice versa. Despite the wide spreading of this epistemic reading of the Heisenberg principle, the Hilbert space formalism clearly shows that the principle has nothing to do with measurements and unavoidable disturbances. The Heisenberg principle is the consequence of a formal feature of quantum mechanics: the existence of incompatible observables, that is, observables represented by non-commuting operators. Non-commutativity is a mathematical feature that formally prevents incompatible observables from having definite values at the same time.

Although non-commutativity is a much deeper feature than mere epistemic uncertainty, it leaves open the possibility that precise values cannot be simultaneously ascribed to incompatible observables due to a limitation of the laws of quantum mechanics itself; the limitation could be remedied by adding some kind of "hidden variables", which would assign definite values to all the observables of a quantum system. But in 1967, Simon Kochen and Ernst Specker (1967) presented a fundamental theorem that proves, in the context of the Hilbert space formalism, that any assignment of a definite value to all the observables of a quantum system leads to a contradiction. In other words, quantum 
mechanics is essentially contextual: definite values can be consistently assigned only in a context of compatible observables. The contextuality of quantum mechanics shows that it is not the case that an electron has a definite momentum, but we do not know its position or vice versa: any assignment of a definite position and a definite momentum is logically forbidden by the very structure of the theory. A quantum "particle" is not an individual in the traditional sense, since it has properties -those represented by its observables- that have no definite value; and this is not a merely epistemic limitation, but an ontological fact described by the theory.

This essentially quantum feature of electrons is usually not considered in quantum chemistry. In many cases, the epistemic reading of the Heisenberg principle still prevails and the Kochen-Specker theorem is ignored:

It is impossible to know simultaneously both the momentum (mass times velocity) and the position of a particle with certainty. In other words, to get a precise measurement of the momentum of a particle we must settle for less precise knowledge of the particle's position, and vice versa (Chang \& Goldsby, 2011, p. 225).

In turn, the electronic density is interpreted as a measure that offers the value of the probability that an electron occupies an infinitesimal region around each point of the physical space. This means that the electron is still conceived as a classical particle that always has a definite but unknown position. Its quantum feature only consists in the fact that its behavior is not governed by classical equations of motion but by an equation, the Schrödinger equation, that determines its position only in a statistical way. This quantum-chemistry picture does not include the essential contextuality of the quantum entities.

\subsection{Non-separability}

According to classical mechanics, if two separate bodies interact, they become separate again when the interaction ends: their further states and properties are completely independent. In order to make this idea more precise, Don Howard formulates a principle of separability, according to which the contents of any two regions of space-time separated by 
a nonvanishing spatiotemporal interval constitute separable physical systems, in the sense that (1) each possesses its own, distinct physical state, and (2) the joint state of the two systems is wholly determined by these separate states (cfr. Howard, 1989, pp. 225-226). This principle is broken in quantum mechanics due to the phenomenon of entanglement. It was Erwin Schrödinger who coined the term 'entanglement' to describe the peculiar connection between quantum systems:

When two systems, of which we know the states by their respective representatives, enter into temporary physical interaction due to known forces between them, and when after a time of mutual influence the systems separate again, then they can no longer be described in the same way as before, viz. by endowing each of them with a representative of its own. I would not call that one but rather the characteristic trait of quantum mechanics, the one that enforces its entire departure from classical lines of thought. By the interaction the two representatives [the quantum states] have become entangled (Schrödinger, 1935, p. 555).

This means that the entangled state of the composite system is not determined by the states of the subsystems.

As a direct consequence of entanglement, the results of measurements on the entangled subsystems of a composite system regularly exhibit patterns of statistical correlation that resist traditional causal explanation. On the other hand, entanglement not only does not disappear when the interaction ends, but also does not decrease with distance or time during the subsequent evolution (for this reason, it must not be confused with the so-called "exchange interaction", which decreases with the distance between particles). This means that, if the subsystems are taken to different laboratories, very far from each other, correlations persist indefinitely. When measurement is conceived in terms of collapse, any measurement that induces collapse on one of the subsystems also induces a simultaneous collapse on the other subsystem, no matter how far the subsystems are from each other.

Quantum non-separability has been interpreted in different ways. Some authors conceive it as the symptom of the non-local nature of reality, that is, of the existence of causal links that can act at-a-distance and simultaneously (see, for example, Maudlin, 1994; Lange, 2002; Berkovitz, 
2016). In this sense, quantum mechanics would violate Einstein's locality, according to which, if $A$ and $B$ are spatially distant entities, then an external influence on $A$ cannot have a simultaneous effect on $B$. Other authors interpret non-separability as the manifestation of ontological holism, according to which the nature and properties of a whole is not determined by the nature and properties of its parts (for example, Teller, 1986; Esfeld, 2001; Healey, 2016). From this viewpoint, what we usually describe as separate measurements on subsystems of an entangled composite system are actually measurements of different properties of a single indivisible whole, not analyzable into parts. If ontologically holist, quantum mechanics would violate Einstein's principle of individuation, according to which the individuation of physical items is determined by their location in space-time: "Without such an assumption of mutually independent existence (the "being-thus") of spatially distant things, an assumption which originates in everyday thought, physical thought in the sense familiar to us would not be possible" (Einstein, 1948, p. 321).

Despite the fact that entanglement and non-separability are usually conceived as essential features of quantum mechanics, in general quantum chemistry operates without considering their ontological consequences. In fact, according to the orbital approximation, the electronic wave function of an atom is computed as the product of the single-electron wave functions for all the electrons of the atom. This move is considered a merely computational resource, an approximation necessary because the exact solution to the Schrödinger equation for situations involving two or more electrons is not achievable. However, it is not sufficiently stressed that this implies to cancel any possibility of entanglement between electrons, which turn out to be treated as classical entities.

Different methods have been developed to obtain the wave function of molecules. Once the presence of the nuclei has already been left aside on the basis of the BOA, the problem consists in describing the behavior of the electrons, which are conceived as independent entities. For instance, the Hartree-Fock method is an iterative method whose starting point is a set of approximate one-electron wave functions: on each electron, the effect of the remaining electrons is approximated by a single averaged effect. The Density Functional Theory, in turn, is based on the electronic density, which is a measure of the probability that an electron occupies an infinitesimal element of space surrounding any given point. By assigning a state to each electron, both under the 
form of one-electron wave functions or of the electronic density, these methods first introduce a sort of reduced state for each electron and then use different approximation strategies designed to solve equations for many-body systems that cannot be solved by analytical methods. In general, the interest is directed to improve the second step, that is, to get better approximations in order to obtain solutions closer to the exact solution. But when the focus is the ontological nature of the electrons, then the first step must be critically assessed: the reduced states of electrons can be viewed as resulting from projections that, by tracing off certain degrees of freedom of the whole composite system, disregard the quantum correlations between the subsystems (see Fortin \& Lombardi, 2014). In other words, from an ontological perspective, quantum chemistry conceives electrons as separately existing individuals: the problem merely consists in solving equations with no analytical solutions in systems composed of many of those individuals. In this sense, the problem is the same as in classical mechanics: the only difference between the two cases lies in the particular forms of the equations to be solved.

\subsection{Indistinguishability}

Another peculiarity of the quantum ontology is the so-called 'indistinguishability' of quantum particles, which is manifested by quantum statistics. In spite of the term by which it is referred to, this is not an epistemic indistinguishability resulting from the limitations of the observer, but rather very a specific characteristic of the quantum objects in their collective behavior.

In their book on identity and individuality in physics, Steven French and Decio Krause (2006) emphasize that the category of individual requires some "principle of individuality". The metaphysical question is, then, what that principle is, which confers individuality to individuals. Different answers have been offered in the history of philosophy, but all of them must explain what it is that makes an individual to be that individual and not another, and that makes the individual the same through the change of its properties.

Let us consider the distribution of two particles, 1 and 2, over two states $|\phi\rangle$ and $|\varphi\rangle$. The question is: how many combinations are possible to obtain the state of the composite system? The classical answer 
is given by the Maxwell-Boltzmann statistics, according to which there are four possible combinations, even in the case that the particles are epistemically indistinguishable: if they are individuals, any principle of individuality, no matter which one, makes particle 1 in $|\phi\rangle$ and particle 2 in $|\varphi\rangle$ a different combination than particle 1 in $|\varphi\rangle$ and particle 2 in $|\phi\rangle$. The peculiarity of quantum statistics is that a permutation of the particles does not lead to a different combination.

Many authors have noticed the serious challenge posed by quantum indistinguishability to the notion of individual. Already in the 60s, Heinz Post (1963) argued that elementary particles must be seen as "nonindividuals" in some sense. Paul Teller (1998) addresses the problem in terms of the traditional notion of "haecceity", that is, what makes an individual to be different from all others in some way that transcends all its properties. According to this author, quantum mechanics provides good reasons to reject any aspect of quantum entities that might do the job of haecceity: "I suggest that belief in haecceities, if only tacit and unacknowledged, plays a crucial role in the felt puzzles about quantum statistics" (Teller, 1998, p. 122). More recently, the idea that quantum "particles" are not individuals acquired a positive content: the quantum ontology is an ontology of properties, without individuals, where quantum systems are mere bundles of properties lacking any individuality (Lombardi \& Castagnino, 2008; da Costa \& Lombardi, 2014; Lombardi \& Dieks, 2016).

Quantum chemistry remains completely alien to these ontological discussions. Indistinguishability is introduced in the computational praxis by means of the so-called 'symmetrization postulate', which puts a restriction on the set of states: non-symmetric states are rendered inaccessible. Certainly, such a restriction is consistent with the ontological view of particles as individuals in a statistical sense: quantum statistics is recovered by regarding those states as possible but never actually realized (French, 1998). However, the restriction to symmetric (bosons) and antisymmetric (fermions) states has an inescapable ad hoc flavor in the context of quantum mechanics. For this reason, some authors claim that the assumption of inaccessible non-symmetric states amounts to the introduction of a surplus structure in the formalism (Redhead \& Teller, 
1992). Furthermore, symmetrization does not overcome the previous obstacles to individuality, that is, contextuality and non-separability.

\section{5.-What ontology does quantum chemistry refer to?}

As explained in the previous section, quantum mechanics, in its standard version, offers a highly puzzling picture of reality, since it challenges the basic ontological category of individual that underlies the classical thought. Quantum systems, such as electrons, protons and neutrons, cannot be considered individuals in the traditional sense. Some of their properties, such as position and velocity, are not always well-defined due to the contextuality of the theory; this feature prevents those systems to be endowed with a precise trajectory over space and time. On the other hand, when two independent quantum systems interact, they cannot be individualized in the composite system anymore, since they constitute a new system, not analyzable into the original components. Finally, quantum statistics gives support to an interpretation of quantum entities as non-individuals.

All these peculiarities make difficult to say what quantum entities really are; nevertheless, what is quite clear is that they are not the individual and localized particles of classical physics. Quantum chemistry, by contrast, although using quantum mechanics as its main computational tool, is still attached to a classical ontological picture: on the basis of the BOA, nuclei behave as classical particles that supply the energetic framework where electrons move; electrons are viewed as minute particles whose precise position and momentum cannot be known with certainty but can be statistically computed by means of the Schrödinger equation. The approximations introduced in the computations are conceived as strategies designed to obtain results in many-body cases, in which analytical solutions cannot be obtained, in the same sense as in a classical theoretical framework. It is worth stressing that this semi-classical picture is not an obstacle for quantum chemistry, whose empirical success makes it one of the most fruitful scientific disciplines at present.

The attachment to a semi-classical picture of reality perhaps explains the positive reception of the so-called 'Quantum Theory of Atoms in Molecules' (QTAIM) in the community of quantum chemists (see, e.g., Matta et al., 2011; Matta, 2013). The QTAIM, proposed by the Canadian quantum chemist Richard Bader (1990), is introduced as the theory 
that supplies the rigorous theoretical foundation, based on quantum mechanics, to chemical notions such as molecular structure and chemical bonding. However, it is still anchored to classical notions when relies on the Born-Oppenheimer approximation and on the notion of electronic density. According to the QTAM, the topology of the electronic density $\rho(r)$ defines chemical concepts. For instance, an atom in a molecule is delimited by a surface of zero-flux of the density gradient field $\nabla \rho(r)$; so, the electronic density of the region limited by that region -the atom-is constant in time. But the idea is appealing because suggests an ontologically classical picture: no electron can cross a zero-flux surface; as the consequence, electrons remain confined to the basin of their respective nuclei and, for this reason, it can be said that each particular electron belongs to a particular atom. On the other hand, the QTAIM allows constructing the molecular graphs of chemical systems, and these representations recover the classical idea of molecular structure in spatial terms, where atoms retain their identity in the molecule and two bonded atoms are linked by means of an accumulation of electronic density.

Summing up, quantum chemistry does not describe a coherent ontology since it combines incompatible concepts coming from classical physics and molecular chemistry on the one hand, and from quantum mechanics on the other. Nevertheless, this fact does not affect its huge empirical success; it is a philosophical issue that can be faced from different philosophical perspectives.

The conclusion about the ontological picture supplied by quantum chemistry is valid to the extent that quantum mechanics is understood as standard quantum mechanics. From a realist viewpoint, it can be stressed that this leaves open the possibility of considering other quantum theories as more appropriate to supply the quantum support to quantum chemistry. The theory that immediately comes to mind is Bohmian mechanics (Bohm, 1952a, 1952b), which appeals to hidden variables to give a complete description of quantum phenomena. In this way, it offers a description that is closer to the principles of classical physics, since subatomic particles are localized individuals and their trajectories can be meaningfully defined (see Tumulka, 2004). The limitation to effectively compute the particles' trajectories is that the 
initial conditions of the quantum system are unknown. For this reason, David Bohm appeals to the statistical reading of the wave function and considers that the initial conditions are distributed with a density of probability given by the square of the wave function. Nevertheless, Bohm stresses that this probability distribution is not due to the ontological nature of quantum systems, but it is merely a consequence of our ignorance of the precise initial conditions of the particle (Bohm, 1952a, p. 171). The affinity between Bohmian mechanics and the ontological picture implicit in quantum chemistry leads us to wonder whether the search for a coherent ontology for quantum chemistry should replace the underlying quantum theory, of course for simplicity reasons not in the computational practice, but from a conceptual viewpoint.

Up to now, it was supposed that quantum chemistry is a scientific discipline that tries to describe certain entities, both in their observable and in their unobservable features and, as a consequence, intends to supply a coherent picture of reality. However, this realist perspective may be rejected from an instrumentalist stance, according to which scientific theories have no representational aspirations and, as a consequence, it is not necessary to search for a coherent ontology that plays the role of their reference. In fact, quantum chemistry can be conceived as a scientific discipline build on the basis of models instead of theories. Although all scientific disciplines use models to apply their theories, from a traditional theory-centered view, models are considered as models of a theory: they depend on theories; a model of a theory cannot lead to contradictions with the theory; the corrections (de-idealizations) introduced in the models must either derive from or be legitimized by the corresponding theory (see, for instance, van Fraassen, 1989; da Costa \& French, 2003; Le Bihan, 2012). Models in quantum chemistry challenge this traditional view since they integrate conceptual elements coming from different and incompatible theoretical domains. Therefore, the independence of quantum-chemical models from classical and quantum theories is not merely provisional and contingent but acquires a conceptual character as long as it is constitutive of the modeling process itself (Lombardi \& Martínez González, 2012; Accorinti \& Martínez González, 2016).

The peculiarity of quantum-chemical models might be appealed to with the purpose of supporting a model-centered view of science, characterized as a "toolbox" conception of scientific theories (Cartwright, Shomar \& Suárez, 1995; Suárez, 1999, 2009; Suárez \& Cartwright, 2008), 
according to which theories are useful tools for model construction. However, this is not the only possible move, and a less radical view makes sense: it can be admitted that the independence of models from theories is not a generic feature of science, but a very peculiar characteristic of quantum chemistry, consequence of the fact that it is what Kostas Gavroglu and Ana Simões (2015) describe as an "inbetween" discipline. According to the authors, after an intricate process of legitimation and search for identity, quantum chemistry is consolidated as an autonomous field of research that takes from physics and chemistry the supplies to build its own scientific practice. From this perspective, the search for a coherent ontology for quantum chemistry loses its meaning to the extent that the practitioners of the discipline are not interested in ontological matters and develop their successful work under the guide of exclusively practical goals.

\section{References}

Accorinti, H. \& Martínez González, J. C. (2016). Acerca de la independencia de los modelos respecto de las teorías. Theoria. Revista de Teoría, Historia y Fundamentos de la Ciencia, 31, 225-245.

Bader, R. (1990). Atoms in Molecules. A Quantum Theory. Oxford: Oxford University Press.

Berkovitz, J. (2016). Action at a Distance in Quantum Mechanics. In E. N. Zalta (ed.), The Stanford Encyclopedia of Philosophy. [URL: http:// plato.stanford.edu/archives/spr2016/entries/qm-action-distance/].

Bohm, D. (1952a). A Suggested Interpretation of the Quantum Theory in Terms of Hidden Variables. I. Physical Review, 85, 166-179. (1952b). A Suggested Interpretation of the Quantum Theory in Terms of Hidden Variables. II. Physical Review, 85, 180-193.

Born, M. \& Oppenheimer, J. R. (1927). On the Quantum Theory of Molecules. Annalen der Physik, 84, 457-484.

Bruer, J. T. (1982). The Classical Limit of Quantum Theory. Synthese, 50, 167-212.

Cartwright, N., Shomar, T. \& Suárez, M. (1995). The Tool Box of Science. En W. Herfel, W. Krajewski, I. Niniiluoto \& R. Wójcicki (eds.), Theories and Models in Scientific Processes. (pp. 137-149). Amsterdam: Rodopi.

Chang, H. (2015). Reductionism and the Relation Between Chemistry and Physics. In T. Arabatzis, J. Renn \& A. Simões (eds.), Relocating 
the History of Science: Essays in Honor of Kostas Gavroglu. (pp. 193209). Dordrecht: Springer.

Chang, R. \& Goldsby, K. (2011). Chemistry. New York: McGraw-Hill.

da Costa, N. \& French, S. (2003). Science and Partial Truth: A Unitary Approach to Models and Scientific Reasoning. Oxford: Oxford University Press.

da Costa, N. \& Lombardi, O. (2014). Quantum Mechanics: Ontology Without Individuals. Foundation of Physics, 44, 1246-1257.

Diestler, D. (2013). Beyond the Born-Oppenheimer Approximation: A Treatment of Electronic Flux Density in Electronically Adiabatic Molecular Processes. Journal of Physical Chemistry A, 117, 4698-4708. Einstein, A. (1948). Quantenmechanik und Wirklichkeit. Dialectica, 2, 320-324.

Esfeld, M. (2001). Holism in Philosophy of Mind and Philosophy of Physics. Dordrecht: Kluwer Academic Publishers.

Fortin, S. \& Lombardi, O. (2014). Partial Traces in Decoherence and in Interpretation: What Do Reduced States Refer to? Foundations of Physics, 44, 426-446.

French, S. (1998). On the Withering Away of Physical Objects. In E. Castellani (ed.), Interpreting Bodies. Classical and Quantum Objects in Modern Physics. (pp. 93-113). Princeton: Princeton University Press. French, S. \& Krause, D. (2006). Identity in Physics: A Historical, Philosophical and Formal Analysis. Oxford: Oxford University Press. Gavroglu, K. \& Simões, A. (2012). Neither Physics nor Chemistry. A History of Quantum Chemistry. Cambridge MA \& London: The MIT Press. (2015). Philosophical Issues in (Sub)Disciplinary Contexts: The Case of Quantum Chemistry. En E. Scerri \& G. Fisher (eds.), Essays in the Philosophy of Chemistry. (pp. 60-79). Oxford: Oxford University Press.

Harré, R. (2009). Trope Theory and the Ontology of Chemistry. Foundations of Chemistry, 11, 93-103.

Healey, R. (2016). Holism and Nonseparability in Physics. In E. N. Zalta (ed.), The Stanford Encyclopedia of Philosophy. [URL = http:// plato. stanford.edu/archives/spr2016/entries/physics-holism/].

Heisenberg, W. (1930). The Physical Principles of the Quantum Theory. Chicago: University of Chicago Press.

Hendry, R. F. (1998). Models and Approximations in Quantum Chemistry. In N. Shanks (ed.), Idealization in Contemporary Physics. (pp. 123-142). Amsterdam-Atlanta: Rodopi. 
(2004). The Physicists, the Chemists, and the Pragmatics of Explanation. Philosophy of Science, 71, 1048-1059.

(2008). Two Conceptions of the Chemical Bond. Philosophy of Science, 75, 909-920.

(2010). Ontological Reduction and Molecular Structure. Studies in History and Philosophy of Modern Physics, 41, 183-191.

Hettema, H. (2009). Explanation and Theory Formation in Quantum Chemistry. Foundations of Chemistry, 11, 145-174.

(2012). Reducing Chemistry to Physics. Limits, Models, Consecuences. Ph.D. dissertation. University of Groningen: Createspace Publishing Platform.

Howard, D. (1989). Holism, Separability and the Metaphysical Implications of the Bell Experiments. En J. Cushing \& E. McMullin (eds.), Philosophical Consequences of Quantum Theory: Reflections on Bell's Theorem. (pp. 224-253). Notre Dame: University of Notre Dame Press.

Kochen, S. \& Specker, E. (1967). The Problem of Hidden Variables in Quantum Mechanics. Journal of Mathematics and Mechanics, 17, 5987.

Labarca, M. \& Lombardi, O. (2010). Why Orbitals Do Not Exist? Foundations of Chemistry, 12, 149-157.

Lange, M. (2002). An Introduction to the Philosophy of Physics. Oxford: Blackwell Publishing.

Le Bihan, S. (2012). Defending the Semantic View: What it takes. European Journal for Philosophy of Science, 2, 249-274.

Lewowicz, L. \& Lombardi, O. (2013). Stuff versus Individuals. Foundations of Chemistry, 15, 65-77.

Lombardi, O. (2014). The Ontological Autonomy of the Chemical World: Facing the Criticisms. In E. Scerri \& L. McIntyre (eds.), Philosophy of Chemistry: Growth of a New Discipline (Boston Studies in the Philosophy and History of Science). (pp. 23-38). Dordrecht: Springer.

Lombardi, O. \& Castagnino, M. (2008). A Modal-Hamiltonian Interpretation of Quantum Mechanics. Studies in History and Philosophy of Modern Physics, 39, 380-443.

(2010). Matters Are Not So Clear on the Physical Side. Foundations of Chemistry, 12, 159-166.

Lombardi, O. and Dieks, D. (2016). Particles in a Quantum Ontology of Properties. In T. Bigaj and C. Wüthrich (eds.), Metaphysics in 
Contemporary Physics (Poznan Studies in the Philosophy of the Sciences and the Humanities). (pp. 123-143). Leiden: Brill-Rodopi.

Lombardi, O. \& Labarca, M. (2005). The Ontological Autonomy of the Chemical World. Foundations of Chemistry, 7, 125-148.

(2006). The Ontological Autonomy of the Chemical World: A Response to Needham. Foundations of Chemistry, 8, 81-92.

Lombardi, O. \& Martínez González, J. C. (2012), Entre mecánica cuántica y estructuras químicas: ¿a qué refiere la química cuántica? Scientiae Studia, 10, 649-670.

Matta, C. F. (2013). Special Issue: Philosophical Aspects and Implications of the Quantum Theory of Atoms in Molecules (QTAIM). Foundations of Chemistry, 15, 245-251.

Matta, C. F., Massa, L., \& Keith, T. A. (2011). Richard F. W. Bader: A True Pioneer. Journal of Physical Chemistry A, 115, 12427-12431.

Maudlin, T. (1994). Quantum Nonlocality and Relativity. Oxford: Blackwell.

Post, H. (1963). Individuality and Physics. Listener, 70, 534-537.

Redhead, M. \& Teller, P. (1992). Particle Labels and the Theory of Indistinguishable Particles in Quantum Mechanics. British Journal for the Philosophy of Science, 43, 201-218.

Rohrlich, F. (1989). The Logic of Reduction: The Case of Gravitation. Foundations of Physics, 19, 1151-1170.

Scerri, E. (2004). Just How $A b$ Initio Is $A b$ Initio Quantum Chemistry? Foundations of Chemistry, 6, 93-116.

Schrödinger, E. (1935). Discussion of Probability Relations between Separated Systems. Proceedings of the Cambridge Philosophical Society, 31, 555-563.

Schummer, J. (2008). Matter versus Form, and Beyond. In K. Ruthenberg \& J. van Brakel (eds.), Stuff. The Nature of Chemical Substances. (pp. 3-18). Würzburg: Königshauen \& Neumann.

Simões, A. \& Gavroglu, K. (2001). Issues in the History of Theoretical and Quantum Chemistry, 1927-1960. In C. Reinhardt (ed.), Chemical Sciences in the 20th Century. Bridging Boundaries. (pp. 51-74). New York: Wiley-VCH.

Suárez, M. (1999). The Role of Models in the Application of Scientific Theories: Epistemological Implications. In M. Morgan \& $\mathrm{M}$. Morrison (eds.), Models as Mediators. (pp. 168-196). Cambridge: Cambridge University Press. 
(2009). Fictions in Science: Philosophical Essays on Modeling and Idealization. New York: Routledge.

Suárez, M. \& Cartwright, N. (2008). Theories: Tools versus Models. Studies in History and Philosophy of Modern Physics, 39, 62-81.

Teller, P. (1998). Quantum Mechanics and Haecceities. In E. Castellani (ed.), Interpreting Bodies. Classical and Quantum Objects in Modern Physics. (pp. 114-141). Princeton: Princeton University Press.

Tumulka, R. (2004). Understanding Bohmian Mechanics: A Dialogue. American Journal of Physics, 9, 1220-1226.

van Brakel, J. (2011). Substances: The Ontology of Chemistry. In R. Hendry, P. Needham \& A. Woody (eds.), Philosophy of Chemistry. (pp. 191-229). Amsterdam: North-Holland.

van Fraassen, B. (1989). Laws and Symmetry. Oxford: Oxford University Press. 